\title{
Hypothyroidism as an Independent Risk Factor for Cardiac Diseases: A Cross-Sectional Study
}

\author{
Dr Gireesh AS. ${ }^{\text {MD }}$
}

\begin{abstract}
Background and Objectives: Hypothyroidism has significant cardiovascular manifestations. Inadequate number of studies and elusive nature of diagnosis of cardiovascular manifestations in hypothyroidism due to less prominence of symptoms and signs; attracted a great deal of investigatory endeavor. This study aims at studying the cardiovascular aftermath in hypothyroid patients by electrocardiography, lipid profile and echocardiography. The completely reversible nature of these complications, after starting treatment is well known.

Methods: 150 Subjects under the study included, out-patient and in-patient hypothyroid cases coming to Sri Siddhartha Medical College and Hospital, Tumkur. Study duration - 1st September 2012 to $31^{\text {st }}$ August 2014. Patients were subjected to history, clinical examination and investigations like thyroid profile, electrocardiography, echocardiography and lipid profile. Data was analyzed using Epiinfo 7.

Results; Out of 150 cases, 97 had clinical hypothyroidism, 53 had subclinical hypothyroidism. 88.67\% cases were females. 54\% were in age group 21-40 yrs. Abnormal ECG in 26\%, abnormal ECHO in 24\%, Diastolic hypertension in 20\%, Dyslipidemia in $34.67 \%$, pericardial effusion in 14\%, Ischemic heart disease in $1.33 \%$ and Anemia in $23.33 \%$ were seen in the study.

Conclusion: The hypothyroid patients present clinically with a myriad of symptoms and signs which are nonspecific. Hence a high index of suspicion is the key for the early diagnosis of hypothyroidism.

The cardiac risk factors seen in the study are in the form of diastolic hypertension, bradycardia, dyslipidemia, $T$ wave changes in ECG, diastolic dysfunction and pericardial effusion in ECHO.

After exclusion of the other risk factors for cardiac disease; various changes seen in hypothyroid cases in this study suggest that hypothyroidism is one of the independent risk factor for cardiac disease.

As the symptoms related to hypothyroidism and cardiac disease overlap, high index of suspicion should be considered for underlying cardiac disease; and these patients should undergo complete cardiac evaluation on diagnosis of hypothyroidism; so as to prevent the catastrophic cardiac outcomes.

Early diagnosis and correction of hypothyroidism is necessary; so that adverse effects on cardiac system can be minimized.

Studies have shown that with treatment of hypothyroidism the cardiovascular risk factors improve.
\end{abstract}

Keywords: Hypothyroidism, ECG, ECHO.

$\mathrm{AF}$

AV

APC

BMI

BMR

CAD

$\mathrm{CCF}$

CNS

COPD

CVS

ECG

ECHO

$\mathrm{EF}$

ELISA

FT4

FNAC

GTT

$\mathrm{Hb}$

HDL

HOCM

\section{Abbrevations}

Atrial fibrillation.

Atrioventricular

Atrial premature contraction

Body mass index

Basal metabolic rate

oronary artery disease.

Congestive cardiac failure

Central nervous system

Chronic obstructive pulmonary disease

Cardio vascular system

Electrocardiography

Echocardiogram

Ejection fraction

Enzyme linked immunosorbant assay

Free T4

Fine needle aspiration cytology

Glucose tolerance test

Haemoglobin

High density lipoprotein

Hypertrophic obstructive cardiomyopathy 


$\begin{array}{ll}\text { IHD } & \text { Ischemic heart disease } \\ \text { I } & \text { Radioactive iodine } \\ \text { IVRT } & \text { Isovolumetric relaxation time } \\ \text { LBBB } & \text { Left bundle branch block } \\ \text { LDL } & \text { Low density lipoprotein } \\ \text { LVDD } & \text { Left ventricular diastolic dysfunction } \\ \text { LVEF } & \text { Left ventricular ejection fraction. } \\ \text { LVH } & \text { Left ventricular hypertrophy. } \\ \text { LVET } & \text { Left ventricle ejection time } \\ \text { LV } & \text { Left ventricle } \\ \text { MHC } & \text { Major Histocompatibility complex } \\ \text { mRNA } & \text { Messenger Ribonucleic acid } \\ \text { MPS } & \text { Mucopolysachharide } \\ \text { PEP } & \text { Pre ejection period } \\ \text { RBBB } & \text { Right bundle branch block } \\ \text { RBS } & \text { Random blood sugar } \\ \text { RIA } & \text { Radio immunoassay } \\ \text { RWMA } & \text { Regional wall motion abnormality } \\ \text { SIADH } & \text { Syndrome of inappropriate ADH production } \\ \text { SCH } & \text { Subclinical hypothyroidism } \\ \text { SD } & \text { Standard deviation } \\ \text { TGL } & \text { Triglyceride } \\ \text { TRH } & \text { Thyroid releasing hormone } \\ \text { TSH } & \text { Thyroid stimulating hormone } \\ \text { USG } & \text { Ultrasonography } \\ \text { VF } & \text { Ventricular fibrillation } \\ \text { VLDL } & \text { Very low density lipoprotein } \\ \text { VPC } & \text { Ventricular premature complexes } \\ \text { VT } & \text { Ventricular tachycardia } \\ & \end{array}$

\section{Aim And Objectives}

\section{Aim}

To study cardiac manifestations in hypothyroidism.

\section{Objectives:}

1. To study the clinical profile of cardiac system in hypothyroidism.

2. To study the various ECG and echocardiographic changes in hypothyroidism.

3. To study the lipid profile in hypothyroidism.

\section{Source of data-}

\section{Methodology}

150 Subjects under the study included, out-patient and in-patient hypothyroid cases coming to Sri Siddhartha Medical College and Hospital, Tumkur.

\section{Method of collection-}

Patients with newly detected and un-controlled hypothyroidism were taken under study. Detailed history, clinical examination, cardiac system examination and investigations were done. Data was analyzed using Epiinfo 7.

\section{Study duration -}

1st September 2012 to $31^{\text {st }}$ August 2014.

\section{Inclusion criteria-}

a. Newly detected hypothyroidism.

b. Old un-controlled hypothyroidism. 


\section{Exclusion criteria-}

Those with congenital heart disease, rheumatic heart disease, diabetes mellitus, hypertension, smoking, alcohol, COPD and severe anemia.

Study design- Cross sectional study.

\section{Investigations}

The following investigations are needed to diagnose hypothyroidism and cardiac diseases.

1. Complete Blood Count: $\mathrm{Hb} \%$, TC, ESR, DC, peripheral Smear.

2. FBS/RBS

3. Blood urea and serum Creatinine.

4. Serum $\mathrm{T}_{3}$

Serum $\mathrm{T}_{4}$

Serum TSH

5. Chest $\mathrm{x}$-ray

6. ECG

7. Lipid profile: Serum Cholesterol, Triglycerides, HDL, LDL, VLDL.

8. Echocardiogram

9. TMT (if needed)

\section{Thyroid profile}

A combination of a raised TSH concentration and a low $\mathrm{T}_{4}$ concentration has great diagnostic value for primary hypothyroidism. In the present study for the measurement of $\mathrm{TSH}, \mathrm{T}_{4}$ and $\mathrm{T}_{3}$ radio-immunoassay was employed.

\section{Electrocardiogram (ECG)}

ECG was recorded in all the patients, in all the 12 standard leads, at a paper speed of $25 \mathrm{~mm}$ per sec. At normal standardization. PR interval of $>0.2$ seconds was taken as prolonged, QRS complexes of less than $5 \mathrm{~mm}$ in limb leads and less than $10 \mathrm{~mm}$ in chest leads were taken as low voltage complexes.

\section{Echocardiogram}

In all the patients in the study echocardiogram was done on Siemens G. Sonoline G. 60S ultrasound imaging system. Cardiac 2D-Mode, M-mode and Doppler. Each case was specifically screened for cardiovascular manifestations like pericardial effusion ventricular dysfunction.

\section{Statistical Methods}

a. Graphical representation of the data.

b. Percentage of data.

c. Measures of central tendency.

Age and Sex distribution:

\section{Results}

Table-4: Distribution of cases according to Age and sex in the study population

\begin{tabular}{|l|c|c|c|c|c|c|}
\hline \multirow{2}{*}{ Age group (yrs) } & \multicolumn{2}{|c|}{ Male } & \multicolumn{2}{c|}{ Female } & \multicolumn{2}{c|}{ Frequency } \\
\cline { 2 - 7 } & $\mathbf{n ( 1 7 )}$ & $\mathbf{\%}$ & $\mathbf{n}(\mathbf{1 3 3})$ & $\mathbf{\%}$ & $\mathbf{n}(\mathbf{1 5 0})$ & $\mathbf{\%}$ \\
\hline $11-20$ & 0 & 0 & 12 & 9 & 12 & 8 \\
\hline $21-30$ & 4 & 23.5 & 38 & 28.5 & 42 & 28 \\
\hline $31-40$ & 4 & 23.5 & 35 & 26.3 & 39 & 26 \\
\hline $41-50$ & 4 & 23.5 & 29 & 21.8 & 33 & 22 \\
\hline $51-60$ & 4 & 23.5 & 13 & 9.8 & 17 & 11.34 \\
\hline $61-70$ & 1 & 5.8 & 6 & 4.5 & 7 & 4.66 \\
\hline Total & 17 & 100 & 133 & 100 & 150 & 100 \\
\hline
\end{tabular}

Maximum numbers of patients were in age group of 21-40yrs - 81(54\%). 


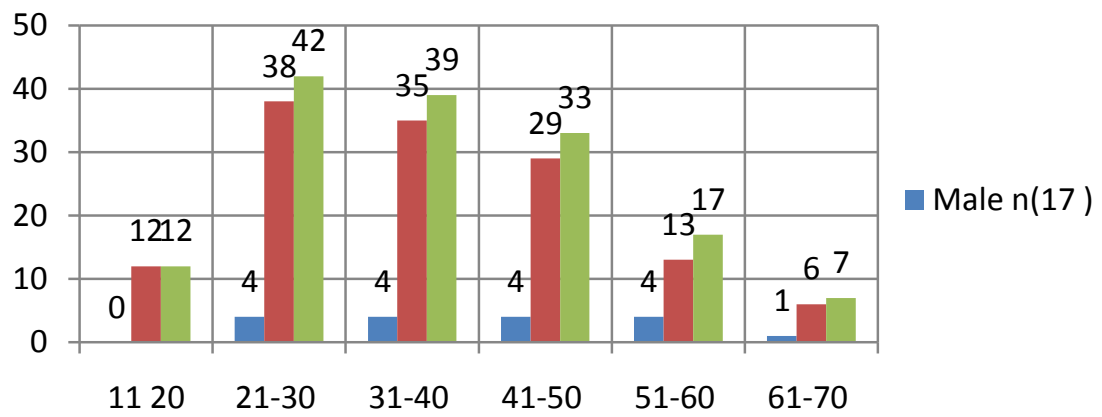

Graph 1: Age and Sex distribution

Table - 4a: Mean age and SD of the cases according to sex

\begin{tabular}{|l|c|c|}
\hline Sex & Mean Age & SD \\
\hline Male & 43.23 & 11.76 \\
\hline Female & 37.49 & 12.89 \\
\hline
\end{tabular}

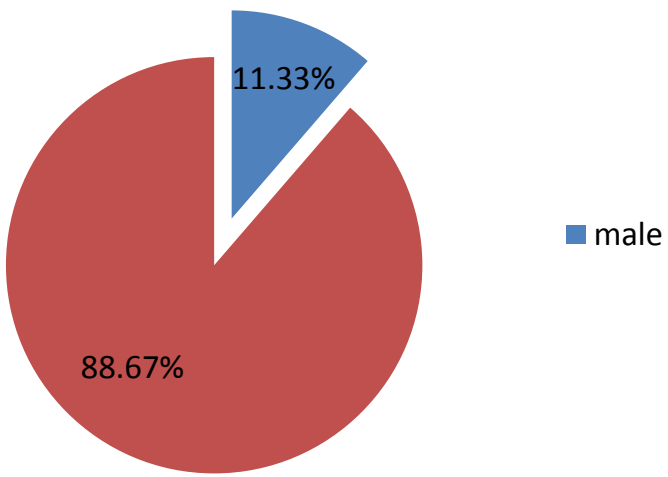

Graph 2: Sex distribution in Sample population

There was overall female preponderance in the study- 133 (88.67\%). Males were 17 (11.67\%).

Table 5: Sex distribution according to clinical and subclinical hypothyroidism

\begin{tabular}{|l|c|c|c|c|c|c|}
\hline \multirow{2}{*}{ Hypothyroidism } & \multicolumn{2}{|c|}{ Male } & \multicolumn{2}{c|}{ Female } & \multicolumn{2}{c|}{ Total } \\
\cline { 2 - 7 } & $\mathbf{n}(\mathbf{1 7})$ & $\mathbf{\%}$ & $\mathbf{n}(\mathbf{1 3 3})$ & $\mathbf{\%}$ & $\mathbf{n}(\mathbf{1 5 0})$ & $\mathbf{\%}$ \\
\hline Clinical Hypothyroidism & 15 & 88.24 & 82 & 61.65 & 97 & 64.67 \\
\hline $\begin{array}{l}\text { Subclinical } \\
\text { Hypothyroidism }\end{array}$ & 2 & 11.76 & 51 & 38.35 & 53 & 35.33 \\
\hline Total & 17 & 100 & 133 & 100 & 150 & 100 \\
\hline
\end{tabular}

97 (64.67\%) patients had clinical hypothyroidism and $53(35.33 \%)$ had subclinical hypothyroidism. 15 $(88.24 \%)$ males had clinical hypothyroidism. In females $82(61.65 \%)$ had clinical and $51(38.35 \%)$ had subclinical hypothyroidism.

Table 6: Sex distribution in hypothyroid cases

\begin{tabular}{|l|c|c|c|c|c|c|}
\hline \multirow{2}{*}{ Hypothyroid } & \multicolumn{2}{|c|}{ Male } & \multicolumn{2}{c|}{ Female } & \multicolumn{2}{c|}{ Total } \\
\cline { 2 - 7 } & $\mathbf{n}(\mathbf{1 7})$ & $\mathbf{\%}$ & $\mathbf{n}(\mathbf{1 3 3})$ & $\mathbf{\%}$ & $\mathbf{n}(\mathbf{1 5 0})$ & \% \\
\hline Newly detected & 12 & 70.6 & 101 & 75.93 & 113 & 75.33 \\
\hline Old under-controlled & 5 & 29.4 & 32 & 24.07 & 37 & 24.67 \\
\hline Total & 17 & 100 & 133 & 100 & 150 & 100 \\
\hline
\end{tabular}

$113(75.33 \%)$ were newly detected hypothyroid cases. 37 cases $(24.67 \%)$ were known hypothyroid under controlled. 


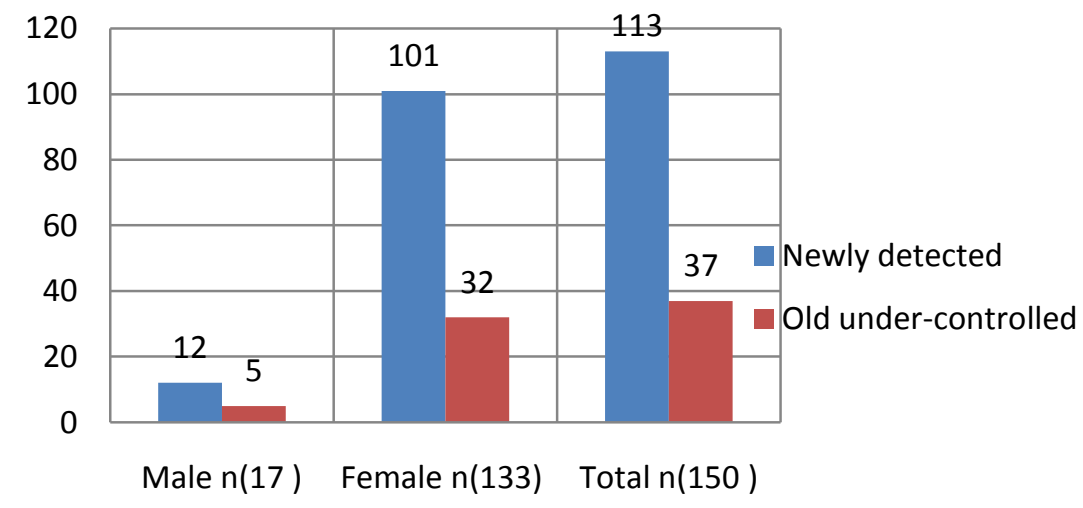

Graph 3: Sex distribution in hypothyroid cases

\section{Symptoms at presentation:}

Table 7: Cardiovascular symptoms at presentation

\begin{tabular}{|l|l|l|l|l|l|l|}
\hline \multirow{2}{*}{ Cardiovascular Symptoms } & \multicolumn{2}{c|}{ Male } & \multicolumn{2}{c|}{ Female } & \multicolumn{2}{c|}{ Total } \\
\cline { 2 - 7 } & $\mathbf{n}(\mathbf{1 7})$ & $\mathbf{\%}$ & $\mathbf{n}(\mathbf{1 3 3})$ & $\mathbf{\%}$ & $\mathbf{n}(\mathbf{1 5 0})$ & $\mathbf{\%}$ \\
\hline Effort Intolerance & 12 & 70.6 & 89 & 66.91 & 101 & 67.33 \\
\hline Swelling of Limbs & 5 & 29.41 & 37 & 27.82 & 42 & 28 \\
\hline Chest Pain & 4 & 23.52 & 24 & 18.04 & 28 & 18.67 \\
\hline Breathlessness & 4 & 23.52 & 21 & 15.8 & 25 & 16.67 \\
\hline Palpitations & 1 & 5.9 & 22 & 16.54 & 23 & 15.33 \\
\hline
\end{tabular}

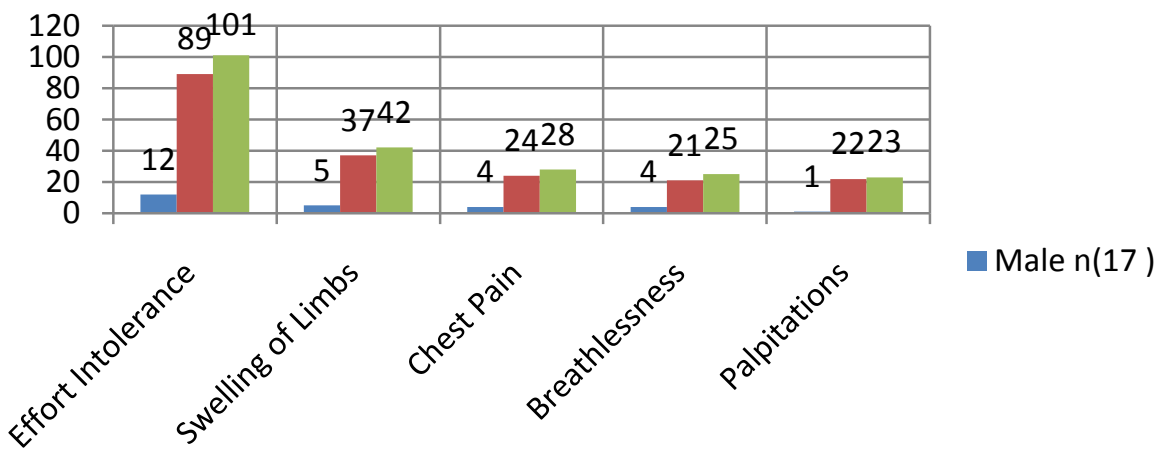

Graph 4: Cardiovascular symptoms at presentation

Most of the patients presented with multiple symptoms. The most common cardiac symptom was effort intolerance $101(67.33 \%)$ in total, other symptoms were swelling of limbs $42(28 \%)$, chest pain $28(18.67 \%)$, breathlessness $25(16.67 \%)$ and palpitations $23(15.33 \%)$.

Table 8: Symptoms related to hypothyroidism

\begin{tabular}{|l|c|c|c|c|c|c|}
\hline \multirow{2}{*}{ Hypothyroid Symptoms } & \multicolumn{2}{|c|}{ Male } & \multicolumn{2}{c|}{ Female } & \multicolumn{2}{c|}{ Total } \\
\cline { 2 - 7 } & $\mathbf{n ( 1 7 )}$ & $\mathbf{\%}$ & $\mathbf{n ( 1 3 3 )}$ & $\mathbf{\%}$ & $\mathbf{n ( 1 5 0})$ & $\%$ \\
\hline Aches \& pains & 7 & 41.18 & 57 & 42.86 & 64 & 42.67 \\
\hline Weight Gain & 8 & 47.06 & 53 & 39.85 & 61 & 40.67 \\
\hline Menorrhagia & 0 & 0 & 48 & 36.1 & 48 & 32 \\
\hline Puffiness of Face & 5 & 29.41 & 36 & 27.06 & 41 & 27.33 \\
\hline Dryness of Skin & 0 & 0 & 25 & 18.8 & 25 & 16.67 \\
\hline
\end{tabular}




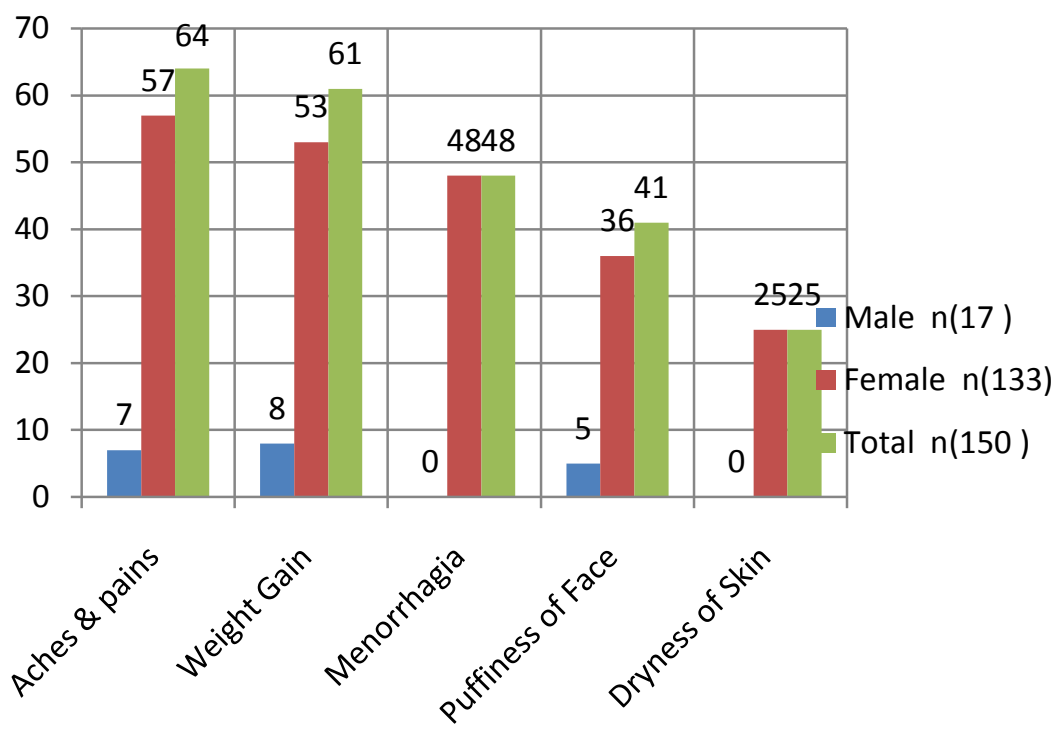

Graph 5: Symptoms related to hypothyroidism

Most patients presented with multiple symptoms. Most common symptoms were aches and pains 64 (42.67\%), weight gain $61(40.67 \%)$, Menorrhagia 48 (32\%), puffiness of face $41(27.33 \%)$ and dryness of skin in 25 $(16.67 \%)$. Subclinical hypothyroid patients presented with very few symptoms.

\section{General Physical examination:}

Table 9: Pulse rate in the study population

\begin{tabular}{|l|l|l|l|l|l|l|}
\hline \multirow{2}{*}{$\begin{array}{l}\text { Pulse rate } \\
\text { Per min }\end{array}$} & \multicolumn{2}{|c|}{ Male } & \multicolumn{2}{c|}{ Female } & \multicolumn{2}{c|}{ Total } \\
\cline { 2 - 7 } & $\mathbf{n}(\mathbf{1 7})$ & \% & $\mathbf{n}(\mathbf{1 3 3})$ & \% & $\mathbf{n}(\mathbf{1 5 0})$ & \% \\
\hline$<60$ & 2 & 11.76 & 4 & 3 & 6 & 4 \\
\hline $60-100$ & 13 & 76.48 & 122 & 91.73 & 135 & 90 \\
\hline$>100$ & 2 & 11.76 & 7 & 5.27 & 9 & 6 \\
\hline
\end{tabular}

\section{Pulse rate beats/min}

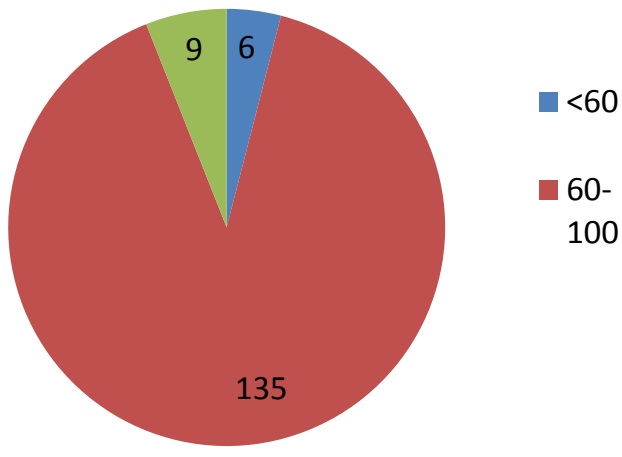

Graph 6: Pulse rate in the study population

Bradycardia ( $<60$ beats/min) was seen in only $6(4 \%)$ cases. Tachycardia ( $>100$ beats/ min) was seen in9 $(6 \%)$. Pulse rate was normal in $135(90 \%)$.

Mean pulse rate is 81.98 . SD is 12.12 Mode is 86 .

Median is 83 .

Table 10: Systolic blood pressure in the study population

\begin{tabular}{|l|l|l|l|l|l|l|}
\hline \multirow{2}{*}{$\begin{array}{l}\text { Systolic BP } \\
\text { mmHg }\end{array}$} & \multicolumn{2}{|c|}{ Male } & \multicolumn{2}{c|}{ Female } & \multicolumn{2}{c|}{ Total } \\
\cline { 2 - 8 } & $\mathbf{n}(\mathbf{1 7})$ & \% & $\mathbf{n}(\mathbf{1 3 3})$ & \% & $\mathbf{n}(\mathbf{1 5 0})$ & \% \\
\hline$<120$ & 3 & 17.65 & 19 & 14.29 & 22 & 14.67 \\
\hline
\end{tabular}




\begin{tabular}{|l|l|l|l|l|l|l|}
\hline $120-139$ & 14 & 82.35 & 112 & 84.21 & 126 & 84 \\
\hline$>140$ & 0 & 0 & 2 & 1.5 & 2 & 1.33 \\
\hline
\end{tabular}

\section{Systolic BP ( $\mathrm{mmHg}$ )}

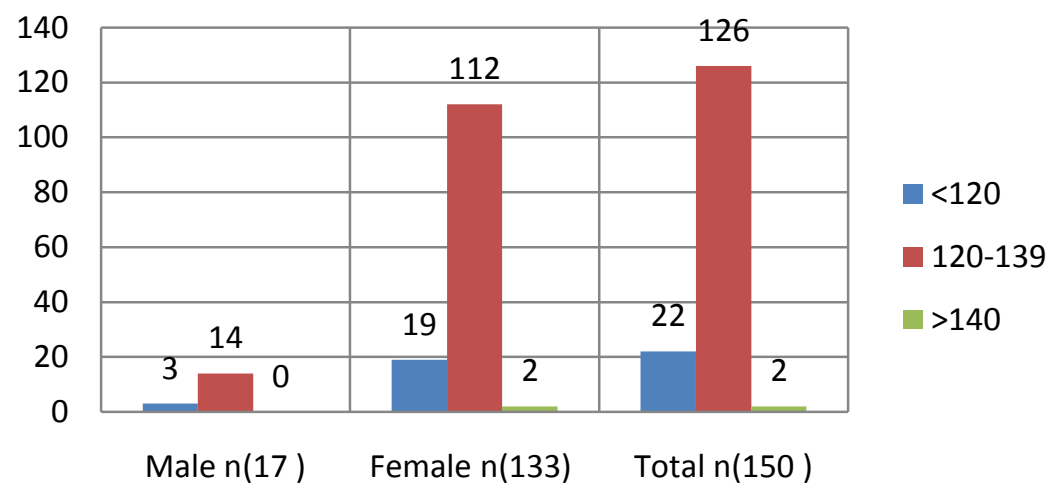

Graph 7: Systolic blood pressure in the study population

Normal systolic blood pressure in $22(14.67 \%)$, pre hypertension in $126(84 \%)$ cases and stage 1 hypertension in $2(1.33 \%)$ cases on presentation according to JNC 7 criteria. $^{35}$

Table 11: Diastolic blood pressure in the study population

\begin{tabular}{|l|l|l|l|l|l|l|}
\hline \multirow{2}{*}{$\begin{array}{l}\text { Diastolic BP } \\
\text { mmHg }\end{array}$} & \multicolumn{2}{|c|}{ Male } & \multicolumn{2}{c|}{ Female } & \multicolumn{2}{c|}{ Total } \\
\cline { 2 - 7 } & $\mathbf{n}(\mathbf{1 7})$ & $\mathbf{\%}$ & $\mathbf{n}(\mathbf{1 3 3})$ & $\mathbf{\%}$ & $\mathbf{n}(\mathbf{1 5 0})$ & \% \\
\hline$<80$ & 3 & 17.65 & 32 & 24.06 & 35 & 23.33 \\
\hline $80-90$ & 11 & 64.7 & 74 & 55.64 & 85 & 56.67 \\
\hline$>90$ & 3 & 17.65 & 27 & 20.3 & 30 & 20 \\
\hline
\end{tabular}

Diastolic BP (mmHg)

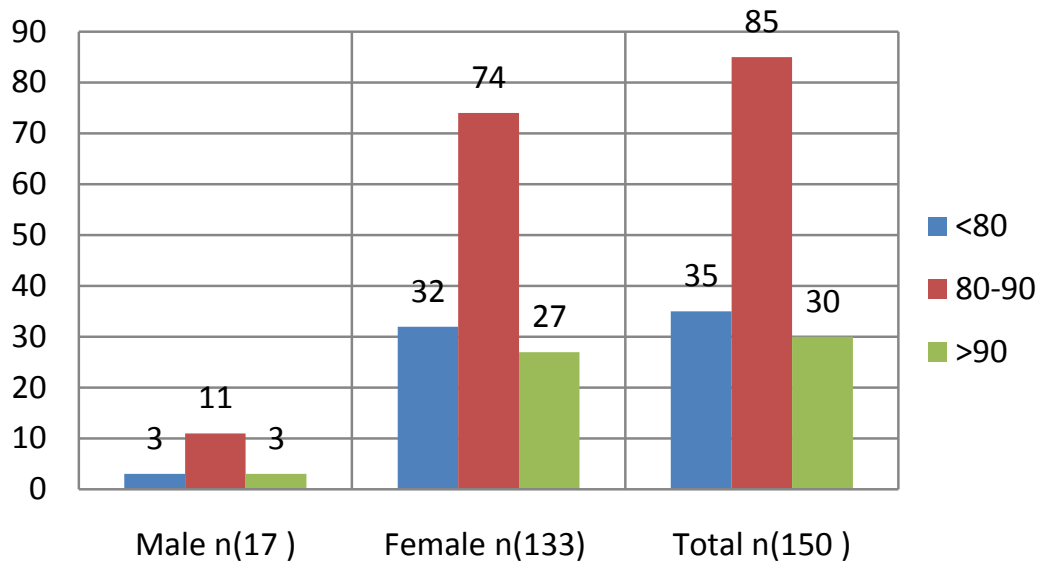

Graph 8: Diastolic blood pressure in the study population

Diastolic blood pressure $>90 \mathrm{mmHg}$ was found in 30 (20\%) of patients.

Table 12: General signs related to hypothyroidism in study population

\begin{tabular}{|l|c|c|c|c|c|c|}
\hline \multirow{2}{*}{ Signs } & \multicolumn{2}{|c|}{ Male } & \multicolumn{2}{c|}{ Female } & \multicolumn{2}{c|}{ Total } \\
\cline { 2 - 7 } & $\mathbf{n}(\mathbf{1 7})$ & $\mathbf{\%}$ & $\mathbf{n}(\mathbf{1 3 3})$ & $\mathbf{\%}$ & $\mathbf{n}(\mathbf{1 5 0})$ & \% \\
\hline BMI >25 Kg/m ${ }^{2}$ & 12 & 70.59 & 70 & 52.63 & 82 & 54.67 \\
\hline Thyromegaly & 3 & 17.65 & 65 & 48.87 & 68 & 45.34 \\
\hline Pallor & 2 & 11.76 & 33 & 24.81 & 35 & 23.33 \\
\hline Dry Skin & 4 & 23.53 & 18 & 13.53 & 22 & 14.67 \\
\hline Edema & 4 & 23.53 & 13 & 9.77 & 17 & 11.34 \\
\hline Delayed ankle jerk & 5 & 29.41 & 5 & 3.76 & 10 & 6.67 \\
\hline
\end{tabular}




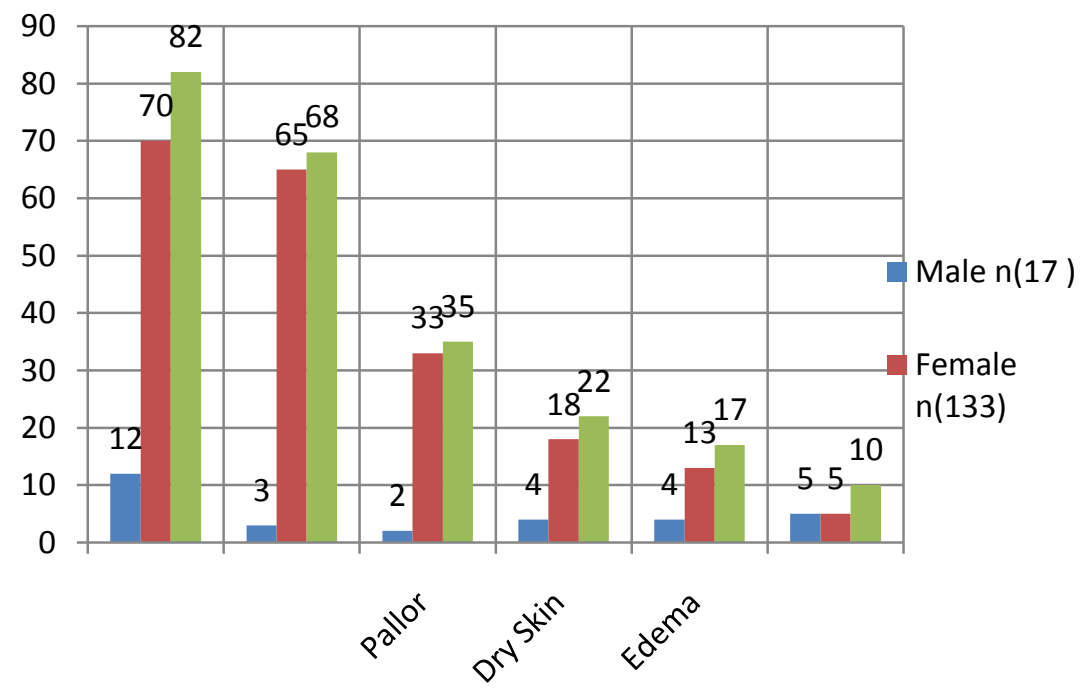

Graph 9: General signs related to hypothyroidism in study population

The most common sign was high body mass index (BMI) $>25 \mathrm{~kg} / \mathrm{m}^{2}$ in $82(54.67 \%)$. Thyromegaly was seen in $68(45.34 \%)$ cases. Pallor in $35(23.33 \%)$, dry skin in $22(14.67 \%)$, edema in $17(11.34 \%)$ and delayed ankle jerk in $10(6.67 \%)$.

\section{Electrocardiographic changes:}

Table 13: Electrocardiographic changes in the study population

\begin{tabular}{|l|c|c|c|c|c|c|}
\hline \multirow{2}{*}{ ECG changes } & \multicolumn{2}{|c|}{ Male } & \multicolumn{2}{c|}{ Female } & \multicolumn{2}{c|}{ Total } \\
\cline { 2 - 7 } & $\mathbf{n}(\mathbf{1 7})$ & $\mathbf{\%}$ & $\mathbf{n}(\mathbf{1 3 3})$ & $\mathbf{\%}$ & $\mathbf{n}(\mathbf{1 5 0})$ & $\mathbf{\%}$ \\
\hline Normal & 9 & 52.94 & 102 & 76.7 & 111 & 74 \\
\hline Bradycardia & 3 & 17.65 & 3 & 2.25 & 6 & 4 \\
\hline Low voltage complex & 1 & 5.89 & 4 & 3 & 5 & 3.34 \\
\hline T wave (Flat / Inverted) & 8 & 47.06 & 29 & 21.8 & 37 & 24.67 \\
\hline ST segment changes & 2 & 11.76 & 3 & 2.25 & 5 & 3.34 \\
\hline
\end{tabular}

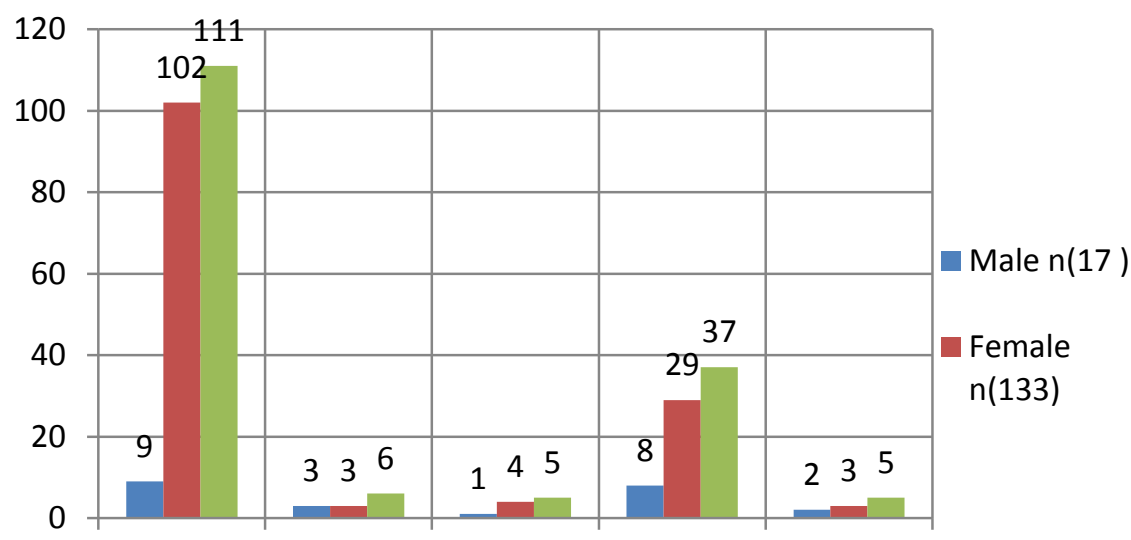

Graph 10: Electrocardiographic changes in the study population

Electrocardiography was normal in $111(74 \%)$ cases and abnormal in 39 (26\%) cases. Most had multiple changes like bradycardia in $6(4 \%)$, low voltage complex in $5(3.34 \%)$, T wave changes in the form of flattening or inversion in $37(24.67 \%)$ and ST segment changes in $5(3.34 \%)$. 


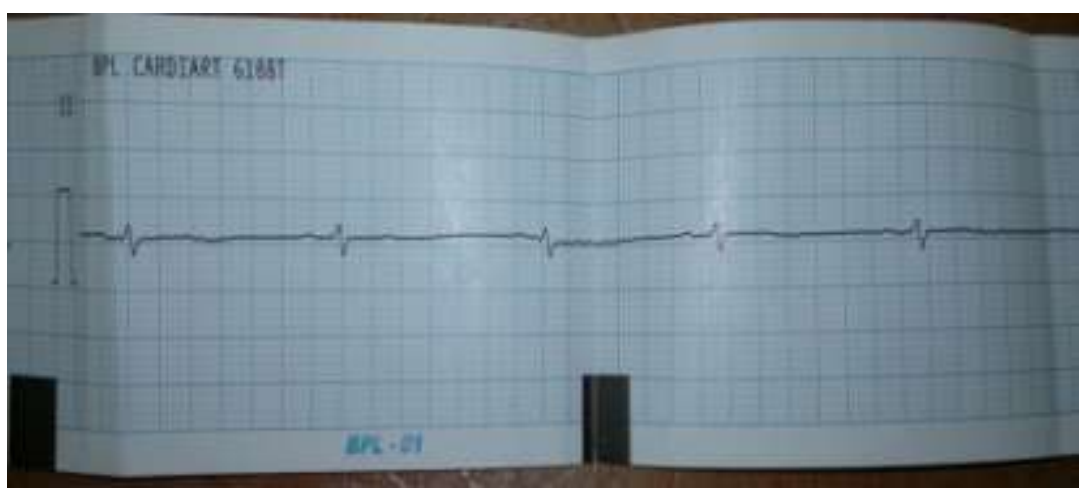

Figure 3: ECG showing Bradycardia

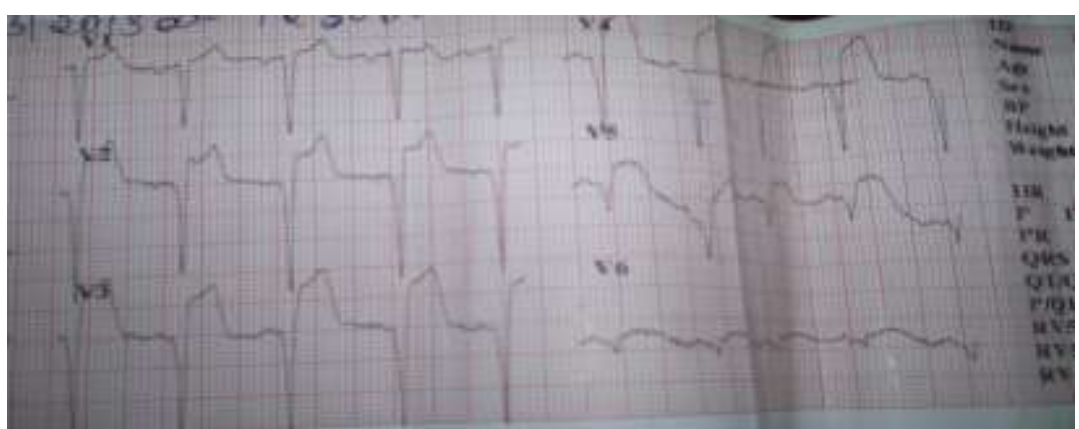

Figure 4: ECG showing anterior wall myocardial infarction in a patient
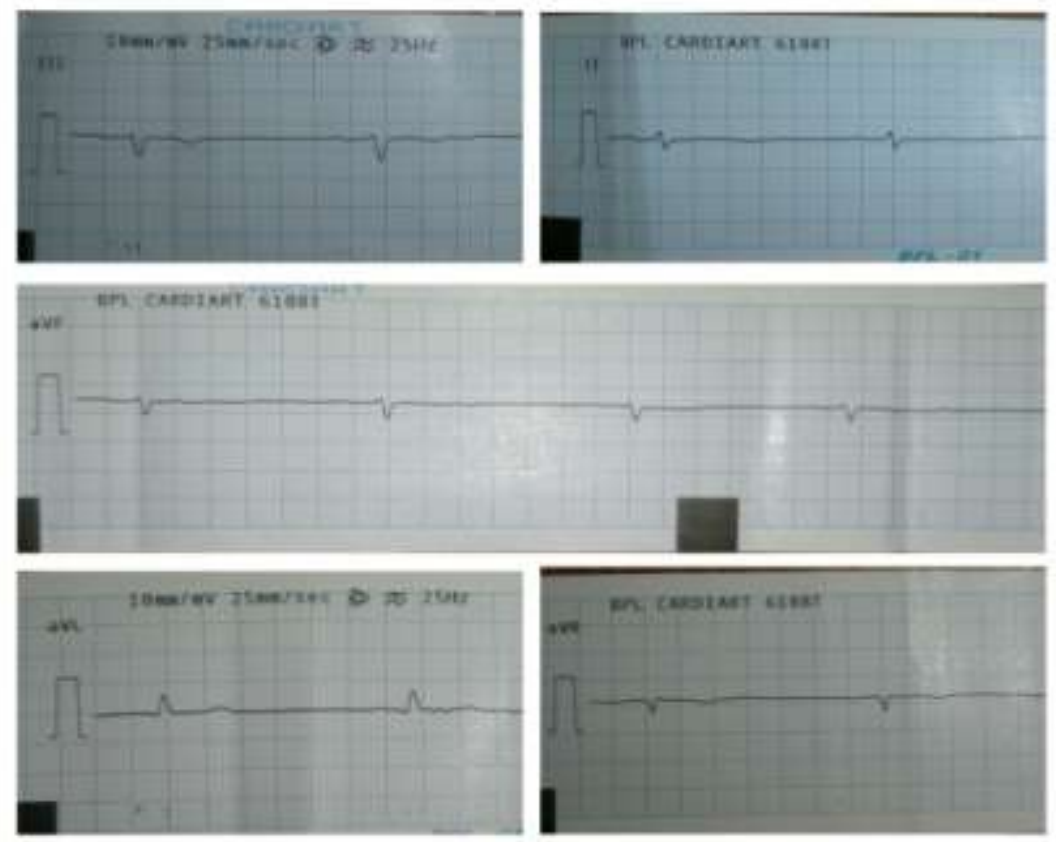

Figure 5: ECG showing low voltage complex in a patient

\section{Echocardiographic changes:}

Table 14: Echocardiographic changes in the study population

\begin{tabular}{|l|c|c|c|c|c|c|}
\hline \multirow{2}{*}{ ECHO changes } & \multicolumn{2}{|c|}{ Male } & \multicolumn{2}{c|}{ Female } & \multicolumn{2}{c|}{ Total } \\
\cline { 2 - 7 } & $\mathbf{n}(\mathbf{1 7})$ & $\mathbf{\%}$ & $\mathbf{n}(\mathbf{1 3 3})$ & $\mathbf{\%}$ & $\mathbf{n}(\mathbf{1 5 0})$ & $\mathbf{\%}$ \\
\hline Normal & 9 & 52.94 & 105 & 78.94 & 114 & 76 \\
\hline LVDD & 5 & 29.41 & 25 & 18.8 & 30 & 20 \\
\hline Pericardial effusion & 4 & 23.53 & 17 & 12.79 & 21 & 14 \\
\hline LVH & 3 & 17.65 & 2 & 1.5 & 5 & 3.34 \\
\hline RWMA & 1 & 5.89 & 2 & 1.5 & 3 & 2 \\
\hline Systolic dysfunction & 1 & 5.89 & 3 & 2.25 & 4 & 2.67 \\
\hline EF $(<50 \%)$ & 1 & 5.89 & 2 & 1.5 & 3 & 2 \\
\hline
\end{tabular}




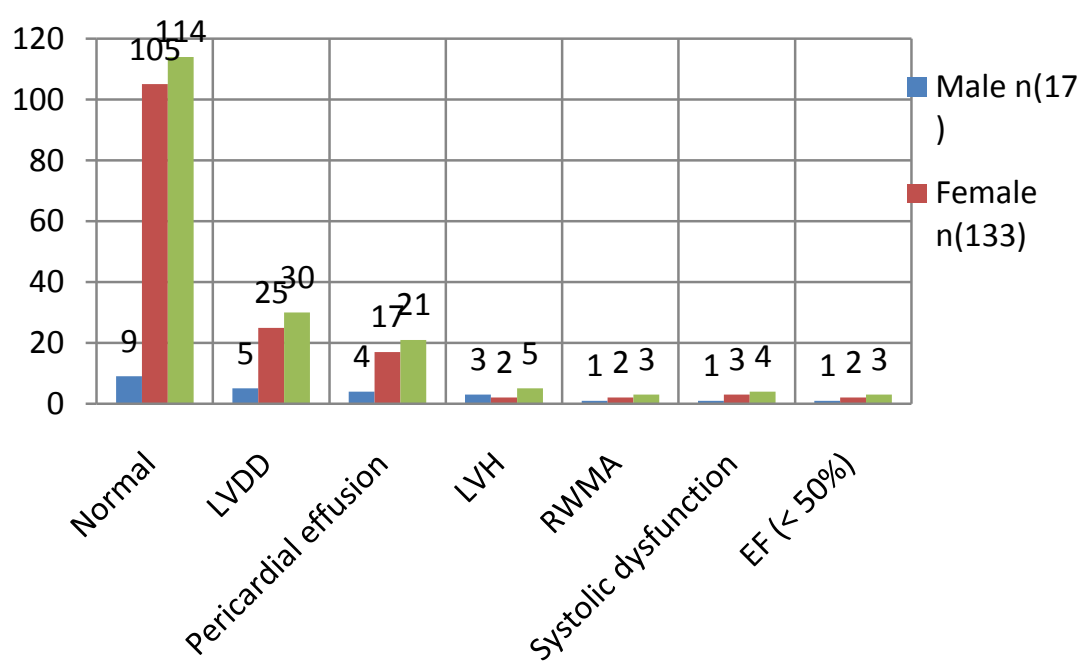

Graph 11: Echocardiographic changes in the study population

Echocardiography was normal in $114(76 \%)$ of cases and abnormal in 36 (24\%) of cases; with most common finding being left ventricular diastolic dysfunction (LVDD) in 30 (20\%), others are pericardial effusion in $21(14 \%)$, left ventricular hypertrophy (LVH) in $5(3.34 \%)$, regional wall motion abnormalities (RWMA) in 3 $(2 \%)$ and systolic dysfunction in $4(2.67 \%)$. EF $<50 \%$ in $3(2 \%)$.

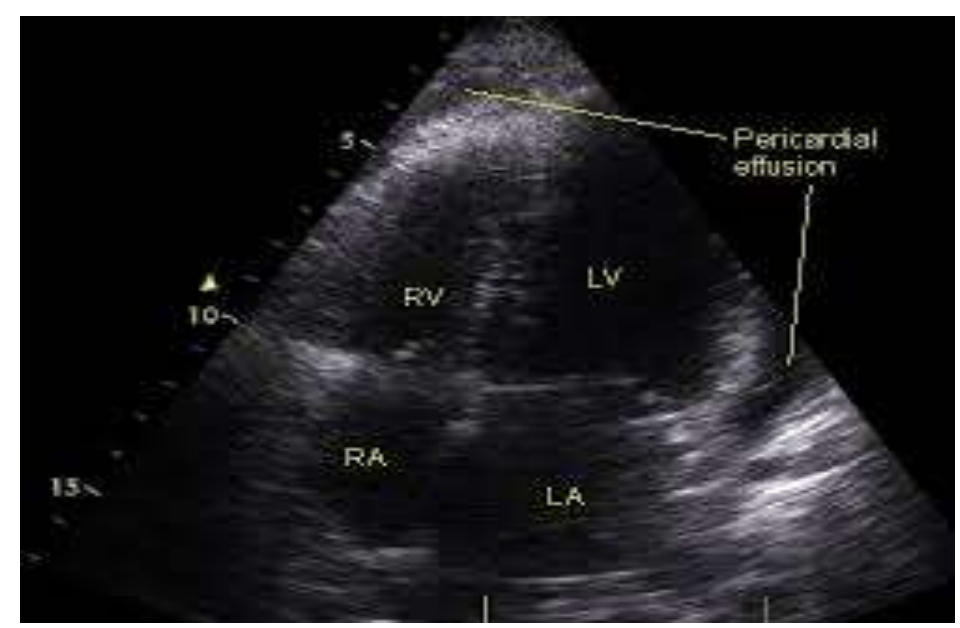

Figure 6: ECHO showing pericardial effusion

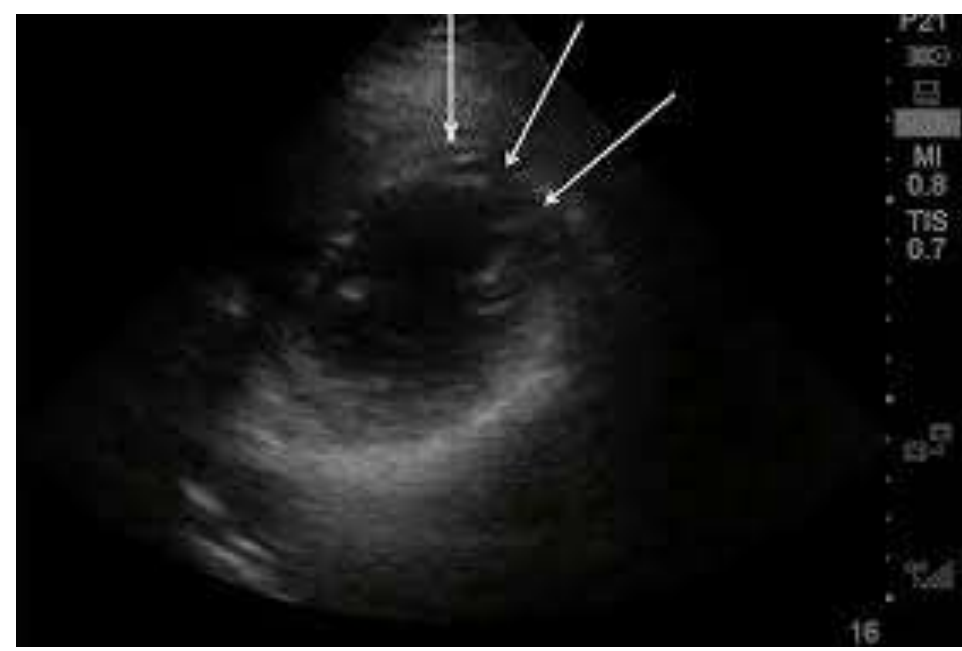

Figure 7: ECHO showing Regional wall motion abnormality 


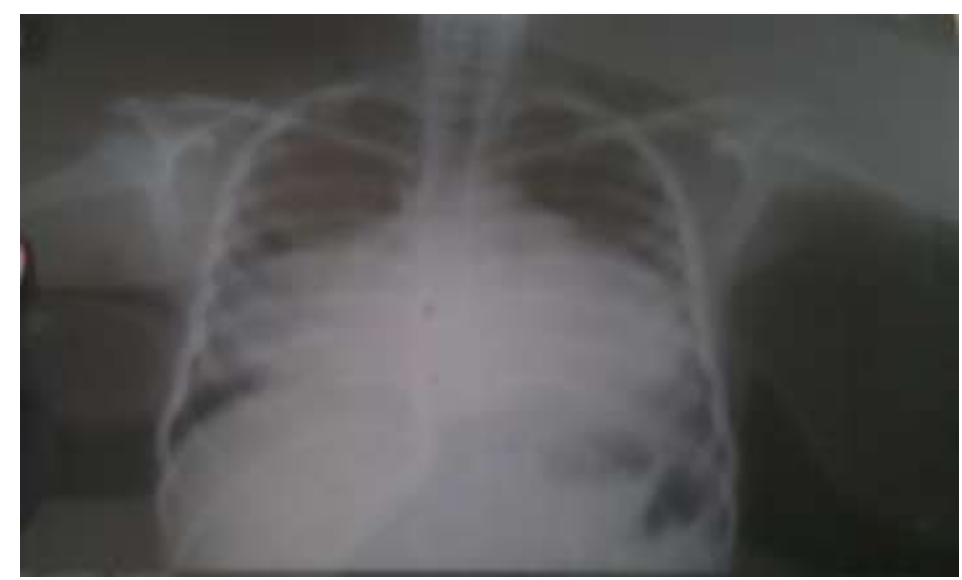

Figure 8: Chest Xray of a patient with hypothyroidism showing "money bag" appearance of cardia due to pericardial effusion

\section{Lipid profile:}

Table 15: Lipid profile changes in the study

\begin{tabular}{|l|l|c|c|c|c|c|c|}
\hline \multicolumn{2}{|l|}{ Lipid Profile (mg/d)) } & \multicolumn{2}{c|}{ Male } & \multicolumn{2}{c|}{ Female } & \multicolumn{2}{c|}{ Total } \\
\cline { 3 - 8 } \multicolumn{2}{l|}{} & $\mathbf{n}(\mathbf{1 7})$ & $\mathbf{\%}$ & $\mathbf{n}(\mathbf{1 3 3})$ & $\mathbf{\%}$ & $\mathbf{n}(\mathbf{1 5 0})$ & $\mathbf{\%}$ \\
\hline Serum Cholesterol & $>200$ & 9 & 53 & 43 & 32.23 & 52 & 34.67 \\
\hline LDL & $>130$ & 8 & 47.05 & 37 & 27.82 & 45 & 30 \\
\hline TGL & $>200$ & 6 & 35.3 & 7 & 5.27 & 13 & 10.67 \\
\hline HDL & $<40$ & 3 & 17.65 & 14 & 10.52 & 17 & 11.34 \\
\hline
\end{tabular}

Dyslipidemia was seen in $52(34.67 \%)$ cases. Most patients had multiple abnormalities. Commonest being hypercholesterolemia in $52(4.67 \%)$; others were high levels of low density lipoprotein (LDL) in 45 (30\%) and hypertriglyceridemia in $13(10.67 \%)$.

\section{Overall changes seen during study:}

Table 16a: Abnormalities seen during study according to sex

\begin{tabular}{|l|c|c|c|c|c|c|}
\hline \multirow{2}{*}{ Changes } & \multicolumn{2}{|c|}{ Male } & \multicolumn{2}{c|}{ Female } & \multicolumn{2}{c|}{ Total } \\
\cline { 2 - 7 } & $\mathbf{n}(\mathbf{1 7})$ & $\mathbf{\%}$ & $\mathbf{n}(\mathbf{1 3 3})$ & $\mathbf{\%}$ & $\mathbf{n}(\mathbf{1 5 0})$ & $\mathbf{\%}$ \\
\hline ECG Changes & 8 & 47.06 & 31 & 23.31 & 39 & 26 \\
\hline ECHO Changes & 8 & 47.06 & 28 & 21.05 & 36 & 24 \\
\hline Dyslipidemia & 10 & 58.82 & 42 & 31.58 & 52 & 34.67 \\
\hline Diastolic Hypertension & 3 & 17.64 & 27 & 20.30 & 30 & 20 \\
\hline IHD & 1 & 5.9 & 1 & 0.75 & 2 & 1.33 \\
\hline CCF & 1 & 5.9 & 4 & 3.01 & 5 & 3.34 \\
\hline Anemia & 2 & 11.8 & 33 & 24.81 & 35 & 23.33 \\
\hline
\end{tabular}

In this study; electrocardiographic changes were seen in 39 (26\%), echocardiographic changes in 36 (24\%), dyslipidemia in $52(34.67 \%)$, diastolic hypertension in $30(20 \%)$, ischemic heart disease in $2(1.33 \%)$, congestive cardiac failure in $5(3.34 \%)$ and anemia in $35(23.33 \%)$.

Table 16b: Abnormalities seen during study according to Serum TSH level

\begin{tabular}{|l|c|c|c|c|c|c|}
\hline \multirow{2}{*}{ Serum TSH $\boldsymbol{\mu I U} / \mathbf{L}$} & \multicolumn{2}{|c|}{$\mathbf{S r} \mathbf{T S H}>\mathbf{1 0} \boldsymbol{\mu I U / L}$} & \multicolumn{2}{c|}{ Sr TSH 6-10 $\boldsymbol{\mu I U} / \mathbf{L}$} & \multicolumn{2}{c|}{ Total } \\
\cline { 2 - 7 } & $\mathbf{n}(\mathbf{9 7})$ & $\mathbf{\%}$ & $\mathbf{n}(\mathbf{5 2})$ & $\mathbf{\%}$ & $\mathbf{n}(\mathbf{1 5 0})$ & $\mathbf{\%}$ \\
\hline ECG Changes & 30 & 30.92 & 9 & 17.30 & 39 & 26 \\
\hline ECHO Changes & 29 & 29.9 & 7 & 13.46 & 36 & 24 \\
\hline Dyslipidemia & 37 & 38.14 & 15 & 28.84 & 52 & 34.67 \\
\hline Diastolic Hypertension & 19 & 19.6 & 11 & 21.15 & 30 & 20 \\
\hline IHD & 2 & 2.06 & 0 & 0 & 2 & 1.33 \\
\hline CCF & 4 & 4.12 & 1 & 1.92 & 5 & 3.34 \\
\hline Anemia & 26 & 26.80 & 9 & 17.30 & 35 & 23.33 \\
\hline
\end{tabular}

The abnormalities seen in the study in relation to serum TSH values $>10 \mu \mathrm{IU} / \mathrm{L}$ (clinical hypothyroidism) were more than that for serum TSH 6-10 $\mu \mathrm{IU} / \mathrm{L}$ (subclinical hypothyroidism) except diastolic hypertension which was $21.15 \%$ in subclinical hypothyroid patients compared to $19.6 \%$ in clinical hypothyroid patients. 
Table 16c: Abnormalities seen in hypothyroid cases during study

\begin{tabular}{|l|l|l|l|l|l|l|}
\hline \multirow{2}{*}{ Changes } & \multicolumn{2}{c|}{$\begin{array}{c}\text { Newly detected } \\
\text { hypothyroid }\end{array}$} & \multicolumn{2}{c|}{$\begin{array}{c}\text { Old under-controlled } \\
\text { hypothyroid }\end{array}$} & \multicolumn{2}{c|}{ Total } \\
\cline { 2 - 7 } & $\mathbf{n}(\mathbf{1 1 3})$ & $\mathbf{\%}$ & $\mathbf{n}(\mathbf{3 7})$ & \% & n(150 ) & \% \\
\hline ECG Changes & 28 & 24.78 & 11 & 29.73 & 39 & 26 \\
\hline ECHO Changes & 31 & 27.43 & 5 & 13.51 & 36 & 24 \\
\hline Dyslipidemia & 39 & 34.51 & 13 & 35.13 & 52 & 34.67 \\
\hline Diastolic Hypertension & 19 & 16.81 & 11 & 29.73 & 30 & 20 \\
\hline IHD & 1 & 0.9 & 1 & 2.7 & 2 & 1.33 \\
\hline CCF & 5 & 4.42 & 0 & 0 & 5 & 3.34 \\
\hline Anemia & 26 & 23 & 9 & 24.32 & 35 & 23.33 \\
\hline
\end{tabular}

Newly detected hypothyroid $\mathrm{n}(113)$

- Old under-controlled hypothyroid $\mathrm{n}(37)$

Total n(150)
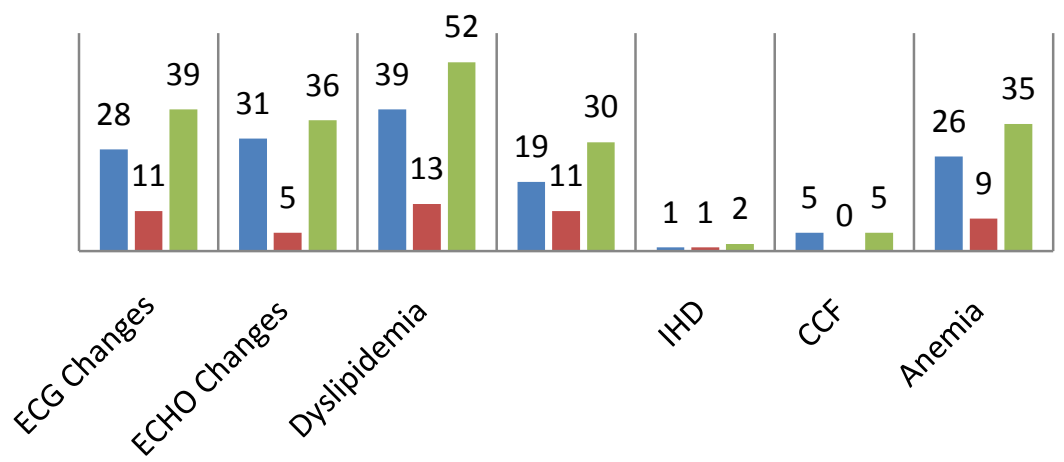

Graph 12: Abnormalities seen in hypothyroid cases during study

As seen from the table the changes seen in the study were more in old under-controlled hypothyroid patients compared to the newly detected hypothyroid cases.

\section{Discussion}

The present study was conducted at Shri Siddhartha Medical College and Hospital, Tumkur. Total numbers of patients were 150. Study period being Sept 2012 to $31^{\text {st }}$ Aug 2014 (24 months).

\section{Age and sex distribution-}

Patients were in the age group of 18-70 yrs. 54\% were in age group of 21-40 yrs. Out of 150, 133 were females and 17 were males.

Table 17: Comparison of percentage of females

\begin{tabular}{|c|c|c|}
\hline $\begin{array}{c}\text { Present Study } \\
(\mathbf{n}=\mathbf{1 5 0})\end{array}$ & $\begin{array}{c}\text { Minshed AJ et al }^{\mathbf{3 6}} \\
\mathbf{2 0 1 0}(\mathbf{n}=\mathbf{3 6})\end{array}$ & $\begin{array}{c}\text { Shah SK et a }^{\mathbf{3}^{37}} \\
\mathbf{2 0 1 3}(\mathbf{n}=\mathbf{3 0})\end{array}$ \\
\hline $88.67 \%$ & $86.1 \%$ & $73.33 \%$ \\
\hline
\end{tabular}

There was a female preponderance seen in this study- $88.67 \%$ compared to other study $86.1 \%$ Minshed $\mathrm{AJ}_{\text {etal }}{ }^{36}$ which was almost same.

Table -18: Comparative study of Symptomatology of hypothyroidism

\begin{tabular}{|c|c|c|c|}
\hline Symptom & $\begin{array}{c}\text { Present Study } \\
(n=150) \%\end{array}$ & Watanakunakorn $1965^{38} \%$ & $\begin{array}{l}\text { Shah SK et } \mathrm{al}^{37} \\
2013(\mathrm{n}=30) \%\end{array}$ \\
\hline Aches and pains & 42.67 & 15.25 & - \\
\hline Weight gain & 40.67 & 47 & 76.66 \\
\hline Menorrhagia & 32 & - & 59 \\
\hline Puffiness of face & 27.33 & 67 & - \\
\hline
\end{tabular}

Weight gain was $40.67 \%$ in present study and $47 \%$ in Watanakunakorn $1965^{38}$. 
Table -19: Comparative study of cardiovascular symptoms in hypothyroidism

\begin{tabular}{|c|c|c|c|}
\hline Symptom & $\begin{array}{c}\text { Present Study } \\
(\mathbf{n}=150) \%\end{array}$ & Watanakunakorn $1965^{38} \%$ & $\begin{array}{c}\text { Minshed AJ et a }^{36} \\
2010(n=36) \%\end{array}$ \\
\hline Effort intolerance & 67.34 & 69.75 & 88.9 \\
\hline Chest pain & 18.67 & 8.25 & - \\
\hline Breathlessness & 16.67 & 12.5 & 75 \\
\hline Swelling of limbs & 28 & - & 11.1 \\
\hline
\end{tabular}

Effort intolerance the most common symptom was $67.34 \%$ in present study and $69.75 \%$ in Watanakunakorn $1965^{38}$.

Table -20: Comparative study of signs in hypothyroidism

\begin{tabular}{|l|c|c|c|}
\hline \multicolumn{1}{|c|}{ SIGN } & $\begin{array}{c}\text { Present Study } \\
(\mathbf{n = 1 5 0 )} \boldsymbol{\%}\end{array}$ & $\begin{array}{c}\text { Shah SK et } \mathbf{~ a l}^{\mathbf{3 7}} \\
\mathbf{2 0 1 3}(\mathbf{n = 3 0}) \mathbf{\%}\end{array}$ & $\begin{array}{c}\text { Minshed AJ et al }^{\mathbf{3 6}} \\
\mathbf{2 0 1 0}(\mathbf{n}=\mathbf{3 6})\end{array}$ \\
\hline Bradycardia & 4 & 40 & 47.2 \\
\hline Diastolic HTN & 20 & 23.33 & 19.5 \\
\hline BMI > 25 Kg/m & & - \\
\hline Thyromegaly & 54.67 & 76.67 & - \\
\hline Edema & 45.34 & 10 & - \\
\hline Pallor & 11.34 & 20 & - \\
\hline
\end{tabular}

Diastolic hypertension was $20 \%$ in present study and $19.5 \%$ in Minshed AJ etal ${ }^{36}$. Edema was $11.34 \%$ in present study and $11.1 \%$ in Minshed $\mathrm{AJ}$ etal ${ }^{36}$.

Table -21: Comparative study of ECG changes

\begin{tabular}{|l|c|c|c|}
\hline Changes & $\begin{array}{c}\text { Present Study } \\
(\mathbf{n = 1 5 0 )} \%\end{array}$ & $\begin{array}{c}\text { Shah SK et } \mathbf{~ a l}^{\mathbf{3 7}} \\
\mathbf{2 0 1 3}(\mathbf{n}=\mathbf{3 0}) \boldsymbol{\%}\end{array}$ & $\begin{array}{c}\text { Minshed AJ et al }^{\mathbf{3 6}} \\
\mathbf{2 0 1 0}(\mathbf{n}=\mathbf{3 6})\end{array}$ \\
\hline Bradycardia & 4 & 40 & 47.2 \\
\hline Low voltage complex & 3.34 & 33.3 & 33.3 \\
\hline T wave changes & 24.67 & - & 27.8 \\
\hline ST segment changes & 3.34 & 26.6 & - \\
\hline
\end{tabular}

$\mathrm{T}$ wave changes were most common $24.67 \%$ in the present study compared to $27.8 \%$ in Minshed AJ etal ${ }^{36}$.

Table -22: Comparative study of ECHO changes

\begin{tabular}{|c|c|c|c|}
\hline Changes & $\begin{array}{c}\text { Present Study } \\
(n=150) \%\end{array}$ & $\begin{array}{l}\text { Shah SK et al }{ }^{37} \\
2013(n=30) \%\end{array}$ & Gupta M.M. et al ${ }^{39} 2000 \%$ \\
\hline LVDD & 20 & 26.67 & - \\
\hline Pericardial effusion & 14 & 26.67 & 15 \\
\hline $\mathrm{LVH}$ & 3.34 & - & - \\
\hline RWMA & 2 & - & - \\
\hline Systolic dysfunction & 2.67 & 6.67 & - \\
\hline
\end{tabular}

Pericardial effusion was $14 \%$ in present study and $15 \%$ in Gupta MM etal ${ }^{39}$.

\section{Lipid profile}

Dyslipidemia was found in 34.67\%. Most common abnormalities were hypercholesterolemia in $34.67 \%$ and high LDL in $10.67 \%$. Similar changes were in study done in NRI General Hospital, Guntur ${ }^{12}$.

Incidence of ischemic heart disease was $1.33 \%$ in present study compared to $11.75 \%$ in Watanakunakorn $1965 .^{38}$

\section{Summary}

This study was conducted on 150 hypothyroid patients with an objective to study cardiovascular changes. Study period being Sept 2012 to $31^{\text {st }}$ Aug 2014.

- Total of 113 cases were newly detected hypothyroid cases and 37 were old uncontrolled hypothyroid cases.

- Patients were in the age group of 18-70 yrs. 54\% were in age group of 21-40 yrs.

- $\quad$ Out of 150, $133(88.67 \%)$ were females and $17(11.33 \%)$ were males.

- $\quad 64.67 \%$ patient had clinical hypothyroidism and $35.33 \%$ had subclinical hypothyroidism in the study.

- The most common cardiac symptom was effort intolerance $67.33 \%$, others were swelling of limbs $28 \%$, chest pain $18.67 \%$, breathlessness in $16.67 \%$ and palpitations in $15.33 \%$.

- Other symptoms were aches and pains $42.67 \%$, weight gain $40.67 \%$ and Menorrhagia $32 \%$.

- Subclinical hypothyroid patients presented with very few symptoms. 
- Diastolic blood pressure $>90 \mathrm{mmHg}$ was found in $20 \%$ of patients. Bradycardia $(<60$ beats $/ \mathrm{min})$ was seen in only $4 \%$ cases.

- $\quad$ BMI $>25 \mathrm{~kg} / \mathrm{m}^{2}$ in $54.67 \%$, Thyromegaly was seen in $45.34 \%$ cases, pallor in $23.33 \%$, dry skin in $14.67 \%$, edema in $11.34 \%$ and delayed ankle jerk in $6.67 \%$.

- Electrocardiography was abnormal in $26 \%$ cases. Most common finding being $\mathrm{T}$ wave changes in the form of flattening or inversion in $24.67 \%$, then bradycardia in $4 \%$, low voltage complex in $3.34 \%$ and ST segment changes in $3.34 \%$.

- Echocardiography was abnormal in $24 \%$ of cases; with most common finding being left ventricular diastolic dysfunction (LVDD) in 20\%, others are pericardial effusion in 14\%, left ventricular hypertrophy (LVH) in 3.34\%, regional wall motion abnormalities (RWMA) in 2\% and systolic dysfunction in $2.67 \%$. $\mathrm{EF}<50 \%$ in $2 \%$.

- Dyslipidemia was seen in $34.67 \%$ cases. Most common being hypercholesterolemia in $34.67 \%$; others were high levels of low density lipoprotein (LDL) in $30 \%$ and hypertriglyceridemia in $10.67 \%$.

- In this study; Ischemic heart disease was found in $1.33 \%$, congestive cardiac failure in $3.34 \%$ and anemia in $23.33 \%$.

- Out of 37 known under-controlled hypothyroid cases; electrocardiographic changes were seen in 11 cases, echocardiographic changes in 5 cases, dyslipidemia in 13 cases, diastolic hypertension in 11 cases, ischemic heart disease in 1 case and anemia in 9 cases.

\section{Conclusion}

- The hypothyroid patients present clinically with a myriad of symptoms and signs which are nonspecific. Hence a high index of suspicion is the key for the early diagnosis of hypothyroidism.

- Most of the symptoms like effort intolerance, lower limb swelling and breathlessness which hypothyroid patients presents with are similar to that of cardiac failure symptoms, hence the cardiac disease is masked and unrecognised.

- The cardiac risk factors seen in the study are in the form of diastolic hypertension, bradycardia, $\mathrm{T}$ wave changes in ECG, diastolic dysfunction and pericardial effusion in ECHO.

- Dyslipidemia seen in the study is also a risk factor for ischemic heart disease.

- After exclusion of the other risk factors for cardiac disease; various changes seen in hypothyroid cases in this study suggest that hypothyroidism is one of the independent risk factor for cardiac disease.

- As the symptoms related to hypothyroidism and cardiac disease overlap, high index of suspicion should be considered for underlying cardiac disease; and these patients should undergo complete cardiac evaluation on diagnosis of hypothyroidism; so as to prevent the catastrophic cardiac outcomes.

- Early diagnosis and correction of hypothyroidism is necessary; so that adverse effects on cardiac system can be minimized.

- Studies have shown that with treatment of hypothyroidism the cardiovascular risk factors improve.

\section{Bibliography}

[1]. Guyton AC and Hall JE. Thyroid Metabolic Hormones. Chapter 76. In: Guyton AC and Hall JE, editors. Text book of Medical Physiology. 11th ed. Pennsylvania: Saunders Elsevier; 2006. p. 931-43.

[2]. Brent GA, Larsen PR and Davies TF. Hypothyroidism and Thyroiditis. Chapter 12. In: Kronenberg HM, Melmed S, Polonsky AS and Larsen PR. Williams Text book of Endocrinology. 11th ed. Philadelphia: Saunders Elsevier; 2007. p. $377-387$.

[3]. Jameson LJ, Weetman AP. Disorders of thyroid gland. Chapter 341. In: Longo DL, Fauci AS, Kasper DL, Hauser SL, Jameson JL and Loscalzo J. Harrison's principles of internal medicine. $18^{\text {th }}$ edition. USA: The McGraw Hill Companies; 2012 . p. $2911-22$.

[4]. Menon UV, Sundaram KR, Unnikrishnan AG, Jayakumar RV, Nair V, Kumar H. High prevalence of undetected thyroid disorders in an iodine sufficient adult south Indian population. J Indian Med Assoc. 2009; 107: 72-7.

[5]. Klein I and Danzi S. Thyroid Disease and the Heart. Circulation. 2007; 116: 1725-35.

[6]. Boelaert K, Franklyn JA. Thyroid hormone in health and disease. J Endocrinol 2005; 187(1): 1-15.

[7]. Reginad H, Maurice FS. Hypothyroidism: Clinical features and complications. Clinics in Endocrinology and metabolism. 1979; 8(1): 29-38.

[8]. Caroline GP Roberts, Paul W. Ladenson. Hypothyroidism. Lancet. 2004; 363: 793-803.

[9]. Kerber E, Richard, Barry S. Echocardiographic Evaluation of Pericardial Effusion in Myxoedema. Circulation. 1975; 52 : 823-827.

[10]. Park YJ, Lee YJ, Choi SI, Chun EJ, Jang HC and Chang HJ. Impact of subclinical hypothyroidism on the coronary artery disease in apparently healthy subjects. European Journal of Endocrinology. 2011; 165: 115-121.

[11]. Razvi S, Weaver JU, Vanderpump MP, and Pearce SH. The Incidence of Ischemic Heart Disease and Mortality in People with Subclinical Hypothyroidism: Reanalysis of the Whickham Survey Cohort. J Clinical Endocrinology Metab. 2010; 95: 1734-1740

[12]. Shekhar R, Chowdary N V S, Das M C, Desai V and Siva P. Prevalence of subclinical hypothyroidism in coastal Andhra Pradesh. Biomedical Research 2011; 22 (4): 471-474.

[13]. Sharma R, Sharma TK, Kaushik GG, Sharma S, Vardey SK and Sinha M. Subclinical hypothyroidism and its association with cardiovascular risk factors. Clin Lab. 2011; 57(9-10):719-24.

[14]. Berlcovitz BK, Collins P. Neck-Thyroid gland. Chapter 31. In: Drake RL, Vogl W and Mitchell AW. Gray's Anatomy. 39 Edition. Philadelphia: Churchill Livingstone; 2005: p. 560-564.

[15]. Serafino F, Emiliano A. Palmieri, et al. Effects of Thyroid Hormone on the cardio-vascular system. Recent progress in Hormone Research. 2004; 59: 31-50. 
[16]. Klein I, Ojamaa K. Thyroid Hormone and the Cardiovascular System. N Engl J. Med. 2001; 334(7): $501-508$.

[17]. Klein I. Endocrine disorders and cardiovascular disease. Chapter 86. In: Braunwald E, et al. The Heart Disease - A textbook of Cardiovascular Medicine. $9^{\text {th }}$ Edition. Philadelphia: WB Saunders Company; 2012: 1833-38.

[18]. Smith OJ, Evans LM, et al. Increased Central Arterial Stiffness in Hypothyroidism. JCEM. 2002; 87(10): 4662-66

[19]. Danzi S, Klein I. Thyroid hormone and the cardiovascular system. Minerva Endocrinol. 2004; 29: 139-50.

[20]. Nikoo MH. Cardiovascular manifestations hypothyroidism. SEMJ. 2001; 2(1):1-14.

[21]. Fletcher AK, Weetman AP. Hypertension and Hypothyroidism. J Hum Hypertension. 1998; 12: 79-82.

[22]. Strachan MW, Walker BR. Endocrine disease. Chapter 20. In: Boon NA, et al. Davidson's Principles and practice of Medicine. 20 edition. Philadelphia: Churchill Livingstone Elsevier; 2002: 750-52.

[23]. Valente M, Desanto C, De Mortine Rosenroll, Dimeos. The direct effect of the thyroid hormone on cardiac chronotopism. Arch Int Physiol Biochem. 1988; 97: 408-31.

[24]. Arem R, Rockey R, Kiefe C, Escalante DA, Rodriguez A. Cardiac systolic and diastolic function at rest and exercise in sub clinical hypothyroidism: effects of thyroid hormone therapy. Thyroid. 1996; 6: 397-402.

[25]. Bonow RO, Udelson JE. Left ventricular diastolic dysfunction as a cause of congestive heart failure. Ann Intern Med. 1992; 117: 502-10.

[26]. Cohen MV, Scalman IC, Sperillo A, Surks ML. Effect of thyroid hormone on left ventricular function in patients treated for thyrotoxicosis. Am j Cardiol. 1981; 48: 33-38.

[27]. Cuocolo A, Sax FL, Brush JE, Maron BJ, Bacharach SL, Bonow RO. Left ventricular hypertrophy and impaired diastolic filling in essential hypertension. Circulation. 1990; 81: 978-86.

[28]. Sichl DB, Chilab HL, Lautensack Belser N, Morgan HE. Faster protein and ribosome synthesis in thyroxine induced hypertrophy of rat heart. Am J of Pysiol. 1985; 285: C309-319.

[29]. Rohrer D, Dillmann WH. Thyroid hormone markedly increases the mRNA coding for sarcoplasmic reticulum Ca ${ }^{++} \mathrm{ATPase}$ in the rat heart. J Biochem. 1998; 213: 6941-6944.

[30]. Duntas LH. Thyroid disease and lipids. Thyroid. 2002; 12(4): 287-93.

[31]. Thompson GR, Soutar AK, Spengel FA, Jadhav A, Gavigan SJ, Myant NB. Defects of receptor-mediated low-density lipoprotein catabolism in homozygous familial hypercholesterolemia and hypothyroidism in vivo. Proc Natl Acad Sci U S A. 1981; 78: 25912595.

[32]. Rush J, Danzi S, Klein I. Role of thyroid disease in the development of statin-induced myopathy. The Endocrinologist. 2006; 16: $279-285$

[33]. Mc Dermott MT. Serum TSH testing is necessary for primary hypothyroidism. J Postgrad Med. 2004; 50: 11

[34]. Shashank R Joshi. Hypothyroidism. The Association of Physicians of India Medicine update. 2005; 63(15): 307-313.

[35]. Chobanian AV, Bakris GL, Black HR, et al. The seventh Report of the Joint National Committee on Prevention, Detection, Evaluation, and Treatment of High Blood Pressure: the JNC 7 report. JAMA. 2003; 289: 2560-72.

[36]. Minshed AJ, Farttoosi AI, Adil S, etal. Cardiovascular Manifestations of Primary Hypothyroidism. The iraqi postgraduate medical journal. 2010; 2: 113-119.

[37]. Shah SK, Kilari M, Shah NK. Cross sectional study of cardiovascular manifestations of hypothyroidism. Journal of Evolution of Medical and Dental Sciences. 2013; 2(27): 5021-29.

[38]. Watanakunakorn C, Robert EH, Titus CE. Myxoedema. Arch Intern Med 1965; 116: 183-89.

[39]. Gupta MM, Doomra M, et al. Heart in hypothyroidism: an Echocardiographic study. JAPI. 2001; $49: 141$. 